\title{
A Review: Flexible Removable Partial Dentures
}

\author{
Dr. Atulya Sharma ${ }^{1}$, Dr. Shashidhara H.S. ${ }^{2}$ \\ ${ }^{\text {I}}$ (P.G. Student, Department of Prosthodontics, College Of Dental Sciences, Davangere, India) \\ ${ }^{2}$ (Professor,Department of Prosthodontics, College Of Dental Sciences, Davangere, India)
}

\begin{abstract}
In an era of implants and fixed prosthesis, removable partial dentures are still a treatment of choice for a wide range of patients and clinicians. As every other treatment option, removable prosthesis also has some disadvantages. But with introduction of flexible partial dentures, many of the disadvantages of conventional acrylic and cast partial dentures are taken care of. This article discusses about the material used in fabrication, indications and compares the flexible partial dentures with other removable partial denture options along with design and insertion technique of the flexible removable partial dentures.
\end{abstract}

\section{Introduction:}

The choice between several treatment options for replacing missing teeth is influenced by clinical, dentist- and patient-immanent factors. Replacement of missing teeth is one of the most important needs for patients attending clinics to restore esthetics and/or function. Many treatment modalities are available for replacing missing teeth; removable partial denture, fixed partial denture or dental implant. Each modality is a possible treatment option and has its own advantages and disadvantages.

Removable partial dentures became popular many decades ago with the introduction of acrylic polymers and chrome cobalt alloys in dentistry. Many patients choose removable partial dentures due to factors ranging from cost to psychology.

Dr. Walter Wright (1937) introduced Polymethyl methacrylate as a denture base material which became the major polymer to be used. Since ages, polymethyl methacrylate (PMMA) has been used to fabricate the dentures. Metal cast / metal frame / metal base dentures are also fabricated to restore the defects. Some of the problems with acrylic are difficult to address, such as insertion in undercut areas, brittleness of methyl methacrylate which leads to fracture, and allergy to methyl methacrylate monomer. In the recent times flexible removable partial dentures have become quite popular. Though search for the ideal option is still on, here is a review of viable option for replacing missing teeth known as flexible dentures.

\section{Material And Its Properties:}

Thermoplastic resins are used for the fabrication of flexible dentures.A thermoplastic is a plastic which becomes pliable or moldable above a specific temperature and returns to a solid state upon cooling. There are different kind of thermoplastic resins available like-

- thermoplastic acetal

- thermoplastic polycarbonate

- thermoplastic acrylic

- thermoplastic nylon

- Because of inherent flexibility of thermoplastic nylon, it is used primarily for flexible tissue borne partial dentures. Chemically, nylons are condensation copolymers formed by reacting equal parts of a diamine and

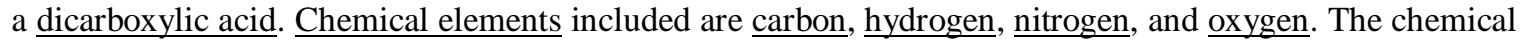
polymerisation reaction is as below:

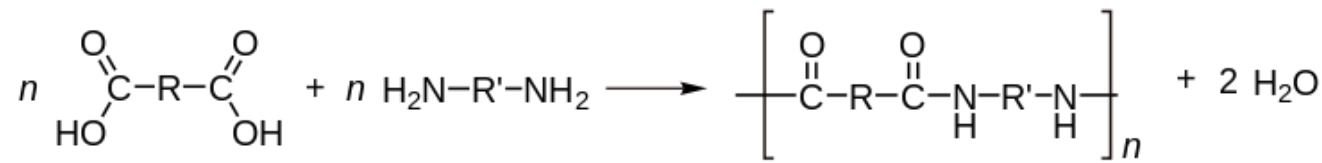

Nylon is a versatile material with characteristics like high physical strength, heat resistance and chemical resistance. It can be easily modified to increase stiffness and wear resistance. Because of its excellent balance of strength, ductility and heat resistance, nylon is the most suitable material available for flexible RPDs. The specific gravity of nylon is 1.14 . The tensile strength is $11000 \mathrm{psi}$ and the flexural strength is $16000 \mathrm{psi}$.[1] When compared with PMMA, valplast (the most commonly used thermoplastic nylon) has higher transverse strength $(117.22 \pm 37.80 \mathrm{MPa})$ as well as impact strength $(0.76 \pm 0.03 \mathrm{kN})$.[2] 


\section{Commercially Available:}

The most suitable and commonly used material for fabrication of flexible removable partial dentures is Valplast(Valpalst Int. Corp. USA).

Other flexible materials available areFlexiplast (Bredent Germany), Lucitone FRS, Flexite, Flexite plus, Flexite M.P. , Sun flex, pro flex.

\section{Indications:}

Flexible removable partial dentures are practically indicated in every partial edentulous condition provided the patients are ready to keep a removable appliance in his/her mouth. Flexible partial dentures utilizes the undercuts in the ridge for retention so it is indicated in ridges where bilateral undercuts are present.

Patients having tilted teeth (due to missing adjacent tooth for long time) develop an undercut where rigid partial denture are tough to insert. In such cases flexible partial dentures are a better option.

Apart from this it is indicated in patients with allergy to acrylic monomers as there is almost no free monomers in this material; cases where clasps have to be given in estheticzone like on maxillary canine, cases where economical conditions limit the use of implant and patient does not want FPDs. In patients who are allergic to nickel, flexible partial dentures can solve the problem faced with cast partial dentures.

In patients with large bony exostoses that can not be removed, flexible partial dentures shows good retention.

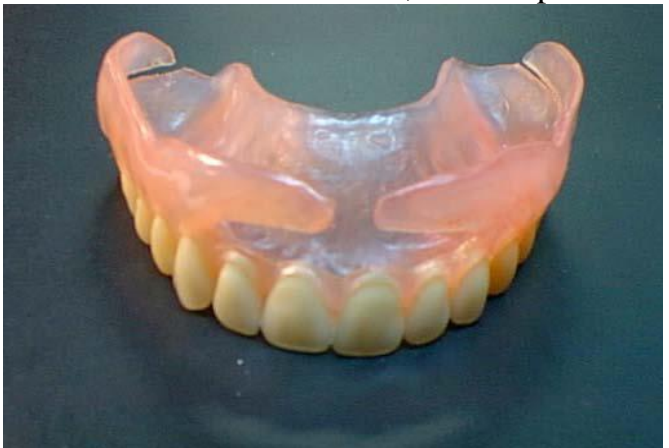

Figure1. Flexibility of the material allows it to engage the undercut beneath the bony exostoses, that is not possible in rigid partial dentutres.

It is also indicated in patients having microstomia, systemic diseases like scleroderma [3] or due to any other reason if there is reduced mouth opening. In such cases flexible partial dentures have shown a good success.

Patients with maxillary tuberosity undercuts often pose challenges in denture fabrication. Flexible denture flanges for patients exhibiting undercut tuberosities can solve this problem. [4]

\section{FRPDs versus Cast partial dentures:}

If we compare the both from the viewpoint of a dentist, time consuming mouth preparations are needed followed by some high skilled surveying techniques in the fabrication of cast partial dentures. [5].

On the part of patients, he/she finds the cast partial dentures much expensive than the FRPDs. Further the cast partial dentures are quite heavy as compared to the FRPDs and hence relatively uneasy to wear.

The next big comparison between the two is esthetics. The thermoplastic nylons are translucent material that allow the underlying tissue to be seen resulting in excellent esthetics. Moreover in FRPDs there are no metal clasps at all. Clasps are also made up of thermoplastic nylons. So clasps can be given on canines without compromising esthetics which is not possible in cast partial dentures.
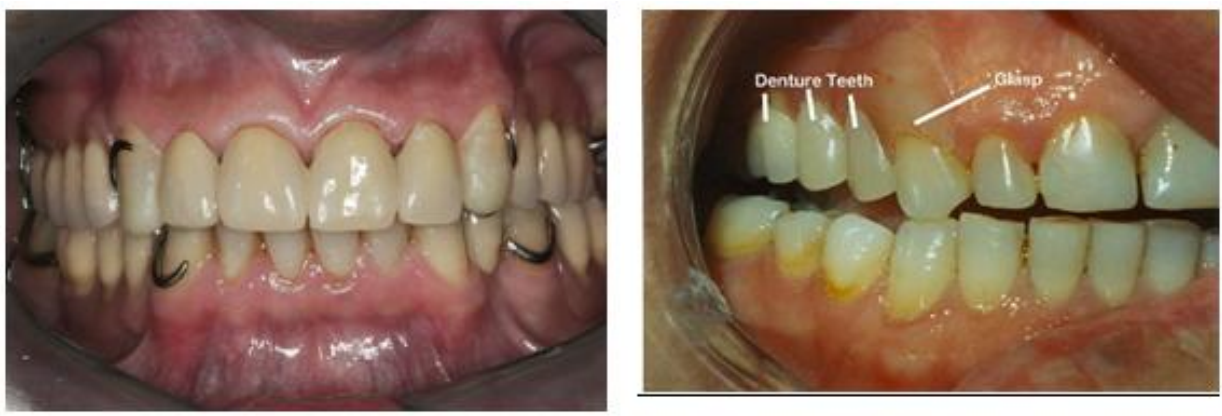

figure2: comparison of esthetics in cast partial dentutre and flexible denture 


\section{FRPDs versus conventional rigid acrylic rpd (temporaries):}

Dentures with thermoplastic nylon do not fracture even if are thrown intentionally from some height whereas patient with full acrylic partial or complete dentuers often visit the dentist with broken or fractured prosthesis as these are brittle. FRPDs show excellent biocompatibility[6] but Acrylic dentuers cause allergic reactions due to free monomer content, high porosity, high water sorption, foul smell and are difficult to insert when undercuts are present. Moreover, laboratory techniques used in the fabrication of FRPDs are more stable and accurate than those used in full acrylic dentures.

Effect on oral mucosa: Flexible dentures exhibit viscoelastic behaviour that lead to improvement in masticatory function and patients comfort compared with hard dentures .Flexible dentures show little effects on the mucosa of denture bearing area and little changes on the mucosa. Denture bearing area of flexible denture are more healthy with less tissues changes compared with traditional acrylic denture. Flexible removable partial dentures can adapt to the shape and movement of mouth and for this reason, these are far more comfortable to wear.[7]

In a study, conducted about the preference among the two types of denture base material; flexible dentures versus conventional acrylic, $100 \%$ patients preferred the flexible dentures over customary methyl methacrylate dentures.[8]

\section{Manipulation And Design:}

The laboratory manipulation of these thermoplastic material is done by injection moulding technique. The acrylic teeth do not bond chemically with the thermoplastic nylon so diatorics are prepared in the artificial tooth to create a mechanical bonding.

A major difference of FRPDs from cast partial dentures is the absence of occlusal rest in the former. Then what about the load distribution among soft and hard tissues. The flexibility of the major connector of the FRPD itself act as a stress breaker. The flexible base of the FRPD floats over the tissue leading to the stress distribution. As there is almost no load on the abutment tooth, no abutment tooth mobility is seen as is seen with rigid cast partial dentures. [6]

There are no metal/wire clasps used in FRPDs. The clasps are also made up of flexible thermoplastic material with excellent esthetics. Different clasp designs are used [5]

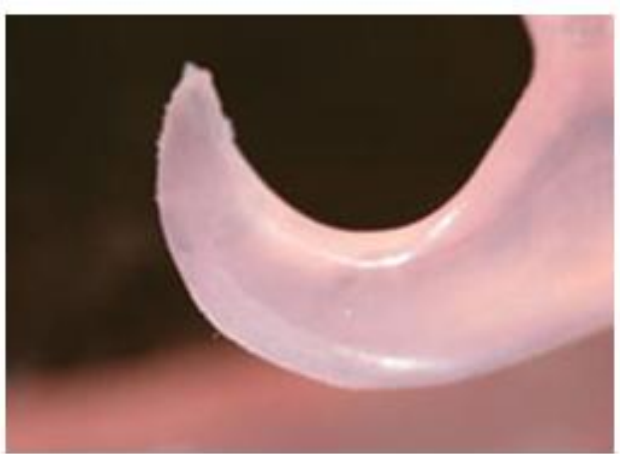

Figure3. Main clasp

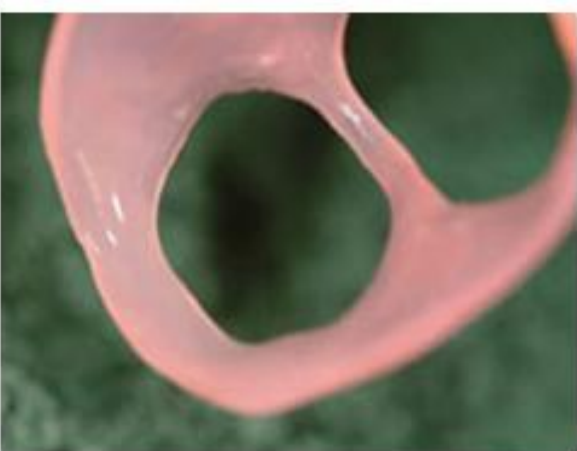

Figure4. Circumfrential clasp

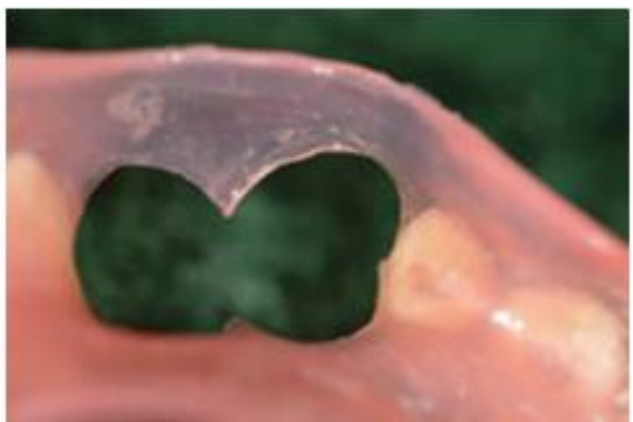

Figure5. continuouscircumfrential clasp 


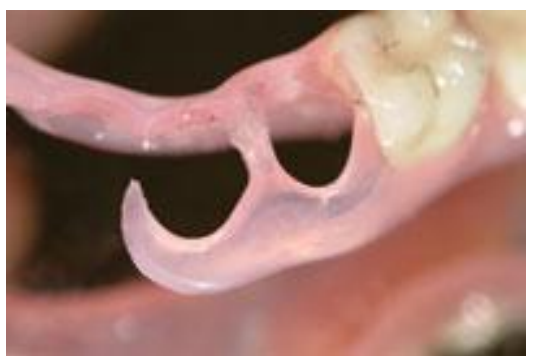

Figure6. Combination clasp ( of main clasp and circumfrential clasp)

If FRPDs are treated same as cast RPD or as acrylic RPDs while insertion and adjustment there are high chances that the prosthesis will fail. The insertion technique for FRPDs is a bit different.Immediately prior to inserting the appliance in patient's mouth, immerse it in very hot tap water. Leave it in the water for about 1 minute, remove and allow to cool to the point where it will be tolerated by the patient. Gently insert in mouth. The hot water permits a smooth initial insertion and good adaptation with the natural tissues in the mouth. If the patient senses any discomfort because of tightness of a clasp, the clasp may be loosened slightly by immersing that area of the partial in hot water and bending the clasp outward. If a clasp requires tightening, bend clasp inward.

If any reduction is needed due to persistent irritation, the resin must be handled differently than acrylic. It is recommend to use green mounted stones (for example, Valplast Green Mounted Point \#202G013, sunflex tool kit). Use a delicate touch with the handpiece rotating between 20,000 and 25,000 rpm in rapid repetitive motion. Then it is smoothened and polished with rubber wheel. The resin will melt if there is prolonged contact with a bur or wheel, so continuously move the instrument over the surface.

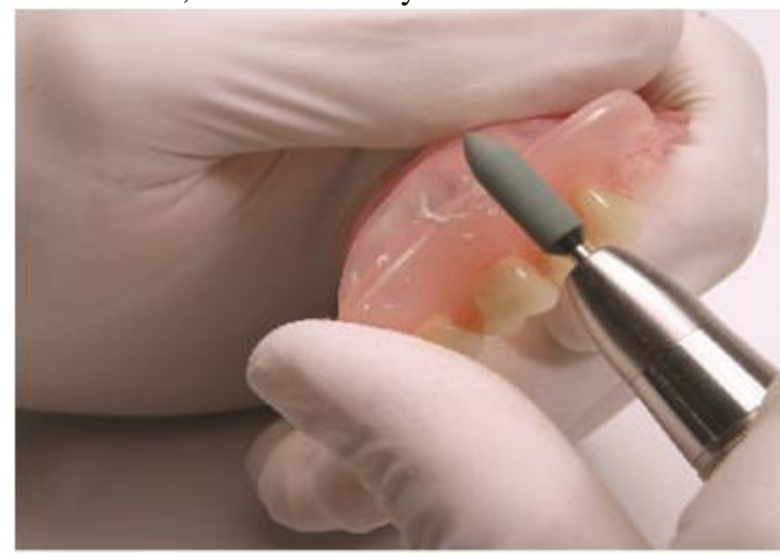

Figure7. using green mounted stone to trim

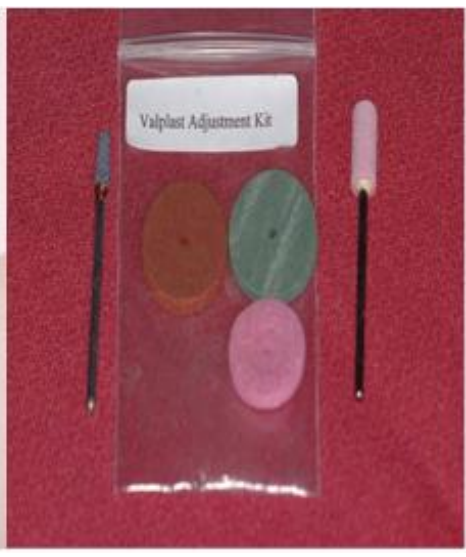

figure8. Valplast adjustment kit

VIII. Disadvantages :

The acrylic teeth are mechanically bonded to thermoplastic nylon. Hence the teeth can come out of the prosthesis.

Chairside techniques for insertion and adjustment are different so need to be learnt. Flexible partial dentures might fail if not inserted properly. Also, one has to purchase separate instruments to adjust/ trim the flexible denture so it might add to the cost.

The elastic modulus of flexible nylon (valplast) is lesser than that of PMMA.[2]

The valplast shows clinically significant chromatic instability, so the flexible removable partial denture might show staining and discoloration with time.[9]

As flexible dentures flex under occlusal load so these can notmaintain the vertical dimension.

When comparing it with cast partial dentures one can criticise the flexible dentures stating that these do not give the patients sense about hot or cold eatables as these are bad conductors.

\section{Conclusion :}

Flexible removable partial dentures are better than other available options especially when there are bilateral undercuts or high esthetic requirements. Proper diagnosis, treatment planning followed by proper insertion techniques can yield good long term results. Though more research work is required to overcome the disadvantages such as poor bonding with acrylic teeth and chromatic staining,the flexible partial dentures appear to have a greater role to play in future. 


\section{References:}

[1]. Negrutiu M, Sinescu C, Romanu M, Pop D, Lakatos S. Thermoplastic resins for flexible framework removable partial dentures. TMJ (timisora medical journal) number 3 year 2005

[2]. KoraySoygun DDS, PhD, GirayBolayir DDS, PhD, Ali Boztug MSc, PhD Mechanical and thermal properties of polyamide versus reinforced PMMA denture base materials. J AdvProsthodont 2013;5:153-60.

[3]. Samet N, Tau S, Findler M, Susarla SM, Findler M. Flexible, removable partial denture for a patient with systemic sclerosis (scleroderma) and microstomia: a clinical report and a three-year follow-up.

[4]. Lowe LG.Flexible denture flanges for patients exhibiting undercut tuberosities and reduced width of the buccal vestibule: a clinical report. The Journal of Prosthetic Dentistry, 2004;92(2):128-131.

[5]. Paul Kaplan, MSci, DDS, MSD Flexible Removable Partial Dentures: Design and Clasp Concepts. Nov. 2008, Dentistrytoday

[6]. 6.Dr.Sunitha N Shamnur1, Dr.Jagadeesh KN1, Dr.Kalavathi SD1, Dr.Kashinath KR "Flexible dentures" - an alternate for rigid dentures? Journal of Dental Sciences \& Research 1:1: Pages $74-79$

[7]. Ghada M. Mustafa BDS, MSc; Mohammed A. Abed AlBaki BDS, MSc.;Sahar A. Naji BDS, MSc; Comparing the Effects of Denture Base Materials on Hygiene of Mucosal Denture Bearing Area. Tikrit Journal for Dental Sciences 1(2013)71-76.

[8]. J. P. Singh, R. K. Dhiman, R. P. S. Bedi, and S. H. GirishFlexible denture base material: A viable alternative to conventional acrylic denture base material. ContempClin Dent. 2011 Oct-Dec; 2(4): 313-317.

[9]. Marcelo Coelho Goiato; Daniela Micheline dos Santos; Marcela Filie Haddad; AldiérisAlvesPesqueira; Effect of accelerated aging on the microhardness and color stability of flexible resins for dentures. Braz. oral res. vol.24 no.1 São Paulo Jan./Mar. 2010. 